\title{
An Empirical Study on Fault Diagnosis for Nonlinear Time Series using Linear Regression Method and FIR Network
}

\author{
Non-Member Muhammad Shafique Shaikh (Muroran Institute of Technology, shafiq@whale.cc.muroran-it.ac.jp) \\ Non-Member Yasuhiko Dote \\ (Muroran Institute of Technology, dote@csse.muroran-it.ac.jp)
}

\begin{abstract}
A fault diagnosis scheme for nonlinear time series is employed for parts and tool breakage diagnosis problems. The fault is first detected from regression lines plotted for the raw time series. Finite Impulse Response (FIR) network is then used to estimate the unknown system for the normal condition data and to filter the abnormal condition data. To confirm and analyze the fault the regression lines are again plotted for the predicted normal and filtered abnormal conditions' data. The results obtained from the above application of fault diagnosis scheme are discussed.
\end{abstract}

Keywords: Signal processing, filtering, fault diagnosis, linear regression, neural network.

\section{Introduction}

For analyzing any set of data first step, in general, is to derive/estimate a mathematical model for it. Once the model is achieved it can be used either for prediction, fault diagnosis, pattern recognition, or pattern classification.

Regression analysis is a statistical technique for modeling and exploring the relationship between variables embed in the unknown system. The relationship between the variables may be linear or nonlinear depending upon the nature of the time series.

The information about a dynamic process is often only partial and incomplete. In many real-world problems, data are masked by noise and some dynamic processes are described by chaotic time series in which the data seem to be random without apparent periodicity [1]. The neural network, being able to acquire knowledge by a learning process and store in massively parallel/ distributed synaptic weights, can solve such complex problems that are intractable.

A kind of neural network, that has short-term memory in the form of tapped delay lines, known as time delay neural network (TDNN) has been used in speech processing [2, 3]. A class of TDNN, that uses finite-duration impulse response (FIR) filters in its synaptic connections between the layers, called as FIR network, are used in time series modeling and prediction $[4,5]$.
In this paper a fault diagnosis scheme $[6,7,8]$ for nonlinear time series is employed. The fault is detected from regression lines of the raw and filtered time series where FIR network is used for modeling and filtering the time series. The described scheme is applied to two fault diagnosis problems using acoustic and vibration data obtained in normal and abnormal conditions from rotating parts of an automobile and a boring machine's tool, respectively.

The paper is organized as follows: Details of linear regression method are given in next section. Section 3 introduces FIR network and its application in this study. Section 4 elaborates the scheme of fault diagnosis using linear regression method and FIR network and its application to acoustic and vibration data. Final remarks are provided in Section 5 .

\section{Linear regression method}

Regression analysis is a statistical technique for modeling and investigating the relationship between two or more variables embed in an unknown system. In the case of simple linear regression a single regressor or predictor $x$ and a response variable $y$ is considered. Therefore,

$$
y=\beta_{0}+\beta_{1} x+\varepsilon
$$


where intercept $\beta_{0}$ and the slope $\beta_{1}$ are unknown regression coefficients, and $\varepsilon$ is a random error with mean zero and variance $\sigma^{2}$. The criterion for estimating the regression coefficients is called as method of least squares. The fitted or estimated regression line from [9] is therefore

$$
\hat{y}=\hat{\beta}_{0}+\hat{\beta}_{1} x
$$

where $\quad \hat{\beta}_{0}=\bar{y}-\hat{\beta}_{1} \bar{x}, \quad \hat{\beta}_{1}=\frac{\sum_{i=1}^{n} y_{i}\left(x_{i}-\bar{x}\right)}{\sum_{i=1}^{n}\left(x_{i}-\bar{x}\right)^{2}}, \hat{y}$ is the estimated trend value, $\bar{y}=(1 / n) \sum_{i=1}^{n} y_{1}$, and $\bar{x}=(1 / n) \sum_{i=1}^{n} x_{i}$.

\section{Neural networks (NN) approach}

A typical use of neural network is (nonlinear) regression, where the task is to find a smooth interpolation between points. The main advantage of the neural network is that it enables us to approximate or reconstruct any nonlinear continuous function $F($.$) ; therefore, such a model is$ more general and flexible.

Suppose $d=f(x)$ describes the input-output relation of an unknown system. Let $y_{1}$ denote the output of the neural network produced in response to an input vector $x_{i}$. The difference between $d_{i}$ and $y_{i}$ provide the error signal vector $e_{i}$, as depicted in Fig. 1.

This error signal is used to adjust the free parameters of NN to minimize the squared difference between the outputs of the unknown system and the $N N$ in a statistical sense.

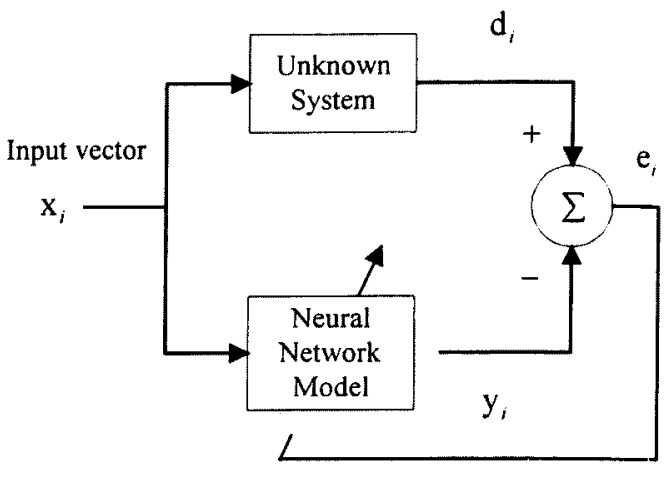

Fig. 1. Block diagram of system identification using neural network.

3.1 Finite-duration impulse response (FIR) filter network

To understand the function of a FIR network, a single neuron extracted from the 1 th layer of an $L$-layer static feedforward neural network adopted from [4] is represented in the Fig. 2. The output of the neuron, $x_{j}^{l+1}$, is taken as a sigmoid function of the weighted sum of its inputs:

$$
x_{j}^{l+1}=f\left(\sum_{i} w_{i, j}^{l} x_{i}^{l}\right)
$$

where $x_{i}^{l}$ and $w_{i, j}^{l}$ are inputs and weights of the neuron, respectively.

A modification of the basic neuron can be accomplished by replacing each static synaptic weight by a FIR linear filter. By FIR we mean that for an input excitation of finite duration, the output of the filter will also be of finite duration. The most basic FIR filter can be modeled with a tapped delay line as illustrated in Fig. 3 . For this filter, the output $y(k)$ corresponds to a weighted sum of past delayed values of the input as described in equation (4). The FIR neuron model is shown in Fig. 4.

$$
y(k)=\sum_{n=0}^{T} w(n) x(k-n)
$$

The feedforward response of the FIR network can be written as, 


$$
x_{j}^{l+1}(k)=f\left(\sum_{i} \mathrm{w}_{i, j}^{l} \cdot \mathrm{x}_{i}^{l}(k)\right)
$$

where $x_{l}^{l+1}(k)$ is the output of a neuron in layer $l$ at time $k$ taken as the sigmoid function of the sum of all filter outputs that feed the neuron. Comparing equations 3 and 5 we may note that the scalars are replaced by vectors. As contrast to standard error backpropagation used in static feedforward neural networks, temporal back-propagation is used in FIR networks. Complete detail of FIR network is given in $[4,10]$.

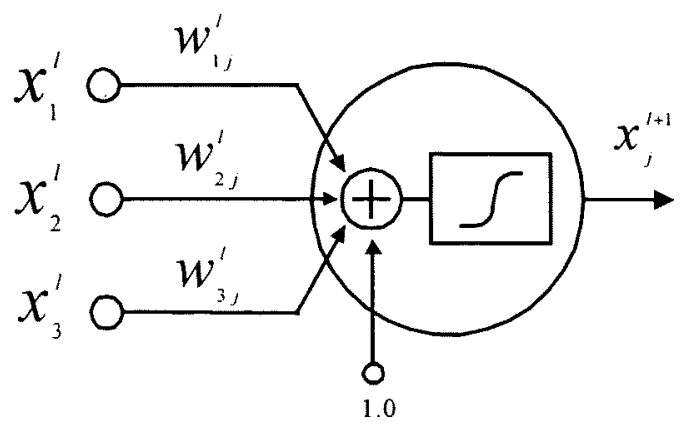

Fig. 2. A static neuron model (feedforward path).
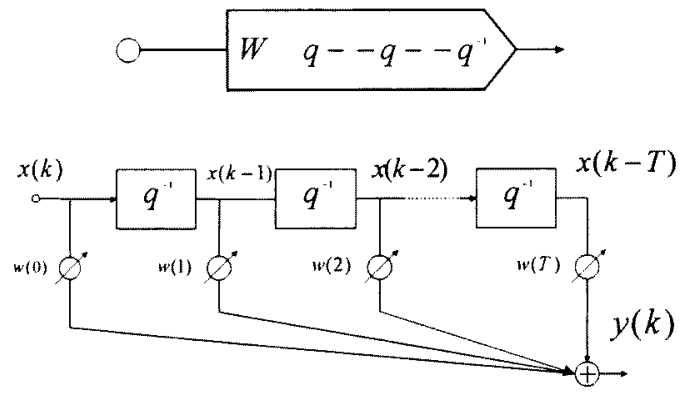

Fig. 3. FIR filter model.

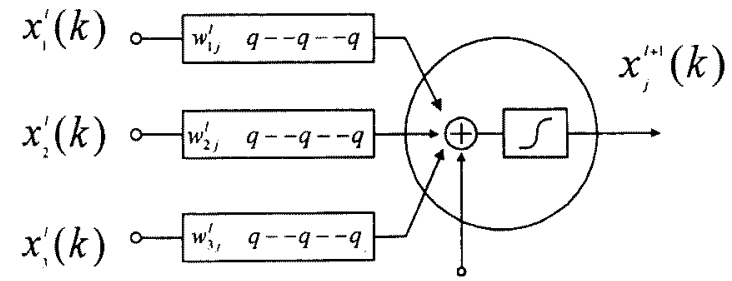

1.0

Fig. 4. A FIR neuron model (feedforward path).

\section{Fault diagnosis scheme}

In the fault diagnosis scheme proposed/described in $[6$, $7,8]$ the fault is first detected from regression lines of the raw time series, recorded in normal and abnormal conditions. Model of the normal condition time series is then estimated using FIR network. The trained network is used for filtering of abnormal condition time series. The fault is further confirmed/analyzed using the regression lines of the predicted normal condition time series and the filtered abnormal condition time series.

The above described fault diagnosis scheme is applied to two fault diagnosis problems, i.e. rotating parts and tool breakage diagnosis, using acoustic data obtained from rotating parts of an automobile and vibration data recorded from a boring tool, respectively.

\subsection{Rotational parts breakage diagnosis}

The above scheme is applied to an automobile's rotating parts' breakage diagnosis problem using acoustic data recorded in normal and abnormal conditions through Integrated Sound Level Meter LA-5110, as shown in Fig. 5. Regression lines (Fig. 6) are first plotted for the raw data, using equation 2 , where a significant difference in the amplitude clearly demonstrates the existence of a fault.

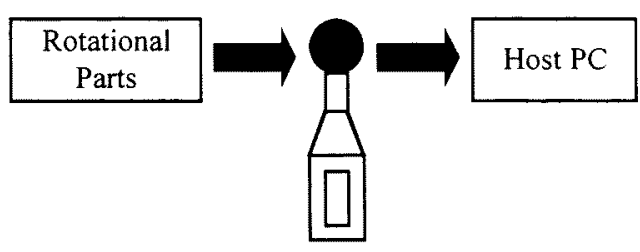

LA-5110

Fig. 5. Rotational parts fault diagnosis experimental setup.

Further study/analysis is carried out after model estimation of the given data. Before model estimation, the two time series are normalized for the range -1 to +1 . Total number of available points is 1000 . Initial 900 points of normalized normal condition data are used for training and the next 100 data points are used for validation. While using FIR networks, selection of number of layers and taps per layer is quite critical. After performing rigorous simulations the best set of number of layers and taps is selected where the mean squared error (MSE) is low. The selected network structure for acoustic 
normal condition data with final MSE is given in Table 1.

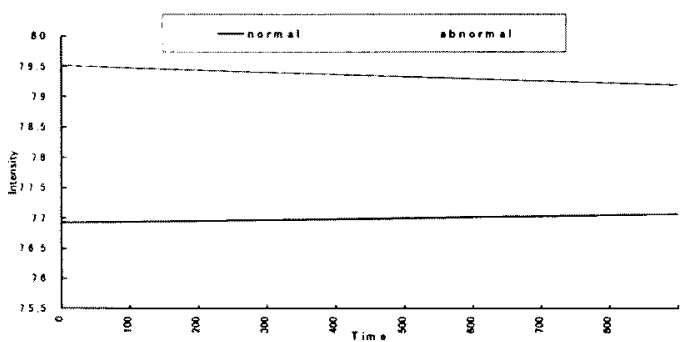

Fig. 6. Regression lines of the acoustic (raw) data.

The MSE pattern for the training set of normal condition data is shown in Fig. 7, where it is noticed that error signal nears zero in about 10 epochs of training. This shows that the selected network has modeled the system quite accurately. The input and output of a trained network for normal condition data are shown in Fig. 8, where the system identification accuracy of the selected FIR network is quite visible.

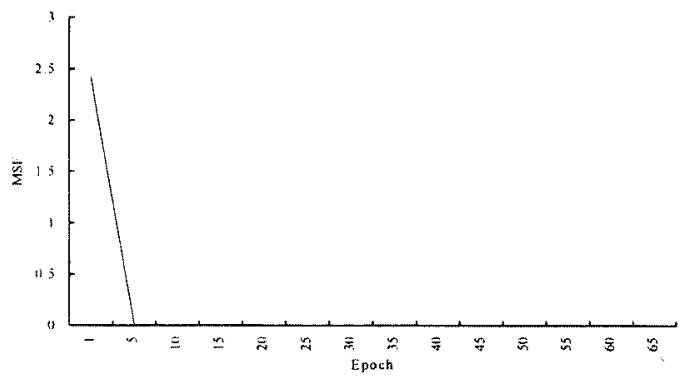

Fig. 7. MSE pattern for training set of acoustic (normal condition) data (900 points).

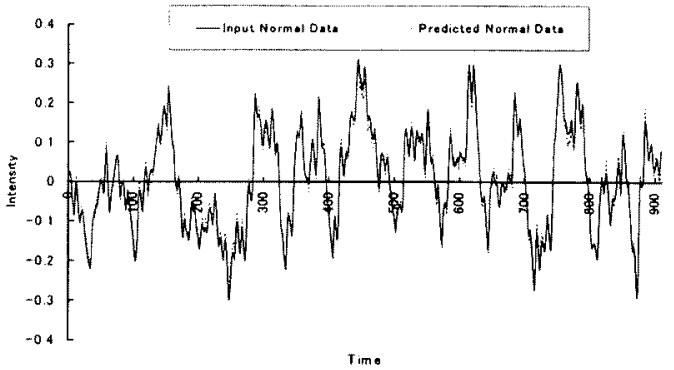

Fig. 8. Input and output of the FIR network trained with normal condition acoustic data.

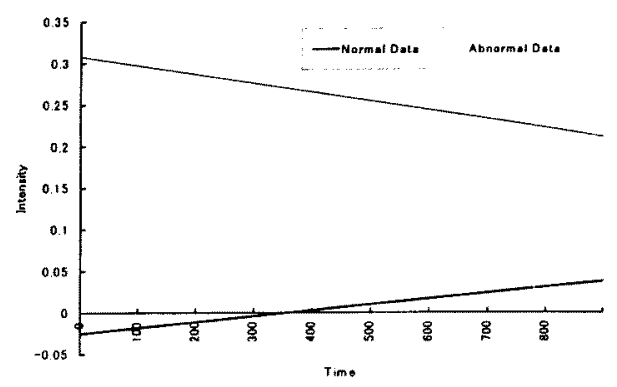

Fig. 9. Regression lines of the predicted normal condition and filtered abnormal condition acoustic data.

The normalized abnormal condition data are then filtered through the trained FIR network. The regression lines for the two time series, i.e. predicted normal condition data and the filtered abnormal condition data, plotted using least square method, are shown in Fig. 9. A significant difference in the two lines (Fig. 9) confirms the existence of the fault that is first detected from the observation of the regression lines of the two original time series (Fig. 6).

\subsection{Boring tool breakage diagrosis}

The described scheme is applied to a boring tool breakage diagnosis problem. An accelerometer (PV-65) is used to acquire the vibration data in normal and abnormal conditions from the boring tool (Fig. 10). As in the previous case regression lines (Fig. 11) are plotted for the raw vibration data. Significant differences in the amplitudes and shapes of these lines clearly exhibit the existence of a fault.

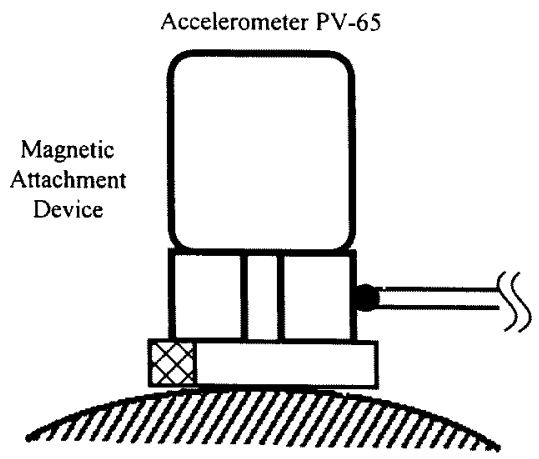

Boring Machine (Pressure Saddle)

Fig. 10. Boring machine fault diagnosis experimental setup 


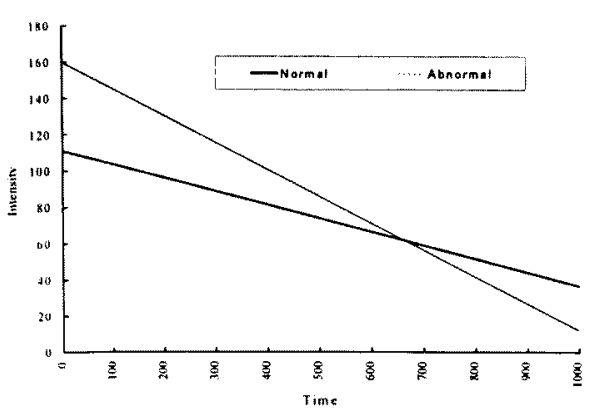

Fig. 11. Regression lines of the vibration (raw) data.

Then the FIR network is used to estimate the model for ormal condition data. Before model estimation both the cormal and abnormal conditions time series are cormalized for the range -1 to +1 . Out of 1000 available lata points, initial 900 points of normal condition data re used for training and the remaining 100 data points or validation. After performing several simulations the rest set of number of layers and taps is selected where he MSE is low and prediction is good. The MSE pattern or the training set of normal condition data is shown in iig. 12. The selected FIR structure for modeling the ıormal condition vibration data is listed in Table 1. It can ve noticed that 18 taps/node are needed at input layer to rain the vibration data as compare to that of 10 aps/node for acoustic data. The input and output of a rained network for normal condition data are shown in i.i. 13, where the model estimation capability of FIR letwork is again evident. The MSE pattern also indicates he accuracy of the model estimation by the selected letwork structure of FIR network.

Table 1. FIR Network structures for acoustic and vibration (normal condition) data.

\begin{tabular}{|c|c|c|}
\hline $\begin{array}{c}\text { Network } \\
\text { Structure }\end{array}$ & Acoustic Data & $\begin{array}{c}\text { Vibration } \\
\text { Data }\end{array}$ \\
\hline Layers & 2 & 2 \\
\hline Input Node & 1 & 1 \\
\hline Input Taps & $10 /$ node & $18 /$ node \\
\hline Hidden Nodes & 30 & 15 \\
\hline Hidden Taps & $3 /$ node & $3 /$ node \\
\hline Output Node & 1 & 1 \\
\hline Epochs & 30,000 & 30,000 \\
\hline MSE & 0.000113998 & 0.00401513 \\
\hline
\end{tabular}

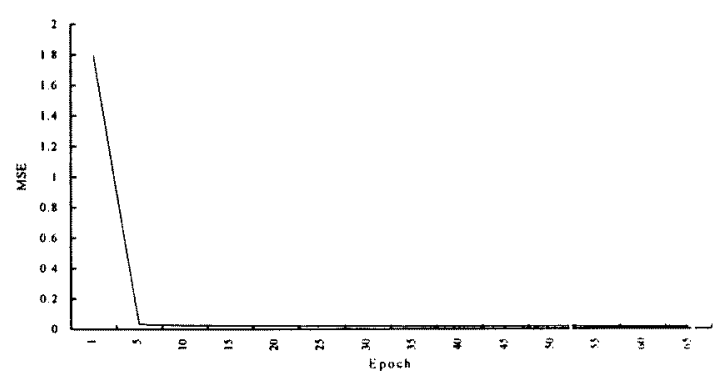

Fig. 12. MSE pattern for training set of vibration (normal condition) data (900 points).

The normalized abnormal condition time series is then fed into the trained FIR network for filtering. The regression lines for the two time series, i.e. predicted normal condition data and filtered abnormal condition data, plotted using least square method, are shown in Fig. 14. A significant difference in the two lines again confirms the existence of the fault that is first detected from the observation of the regression lines of the two original time series (Fig. 11). The behavior and amplitude of the regression lines (Fig. 14) not only confirms the existence of the fault but also increases the sensitivity of the fault detection.

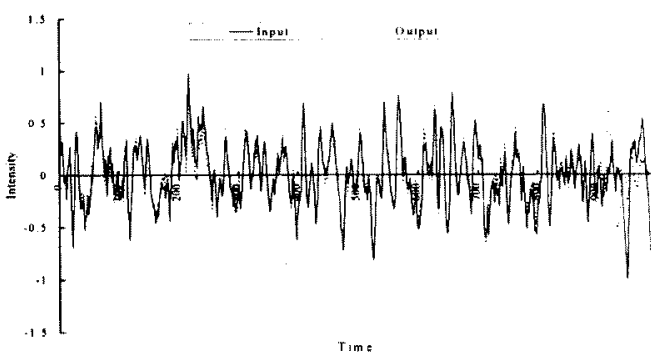

Fig. 13. Input and output of the network trained with normal condition vibration data.

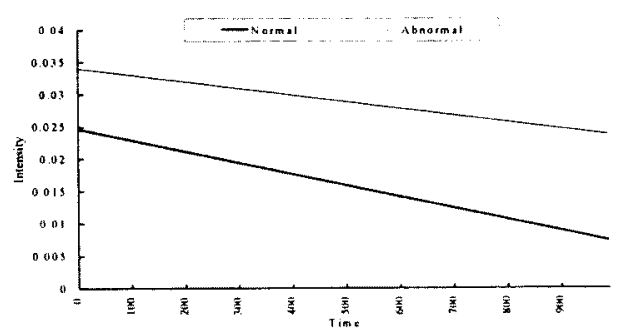

Fig. 14. Regression lines of the predicted normal and filtered abnormal conditions vibration data. 


\section{Conclusion}

The fault diagnosis scheme is applied to two real-world applications using acoustic and vibration data recorded from rotating parts of an automobile and a boring tool in normal and abnormal conditions. Initial fault detection using regressions lines of the raw data are confirmed from the regression lines of the predicted normal and filtered abnormal conditions' data. Hence it can be safely said that the described fault diagnosis scheme is suitable for the given two fault diagnosis problems. The FIR network is found suitable for model estimation of unknown system, which may be suitable for other applications, e.g. pattern classification/recognition, etc.

The selected FIR network structure is good only for the used application. To estimate model for other data fresh simulation would be needed. Preprocessing of the raw data may be helpful in realizing more accurate model.

(Manuscript received Dec. 6, '99, rivised March 1, 2000)

\section{References}

(1) A. Cichocki and R. Unbehauen, Neural Networks for Optimization and Signal Processing, John Wiley \& Sons, New York, 1993, pp. 449.

(2) K. J. Lang and G. E. Hinton, "A Time-Delay Neural Network Architecture for Speech Recognition," Technical Report CMU-CS-88-152, Carnegie-Mellon University, Pittsburgh, PA, 1988.

(3) A. Waibel, T. Hanazawa, G. Hinton, K. Shikano, and K. J. Lang, "Phoneme Recognition Using Time-Delay Neural Networks," IEEE Transactions on Acoustics, Speech, and Signal Processing, Vol. 37, No. 8, 1989, pp. 328-339.

(4) E., Wan, "Time Series Prediction by Using a Connectionist Network with Internal Delay Lines," Time Series Prediction: Forecasting the Future and Understanding the Past (A. S. Weigend and N. A. Gershenfeld, eds.), Addison Wesley, Reading MA, 1993, pp. 195-217.

(5) W. Saad, D. V. Prokhorov, and D. C. Wunsch, "Comparative Study of Stock Trend Prediction Using Time Delay, Recurrent, and Probabilistic Neural Networks," IEEE Transactions on Neural Networks, Vol. 9, No. 6, 1998, pp. 1456-1470.

(6) M. S. Shaikh, D. Akhmetov, and Y. Dote, "Fault Diagnosis from Nonlinear Time Series using Time Delay Neural Network", Proc. of Int. Workshop on Soft Computing in Industry '99 (IWSCI'99), Muroran, Japan, 16-18 June 1999, pp. 362-367.
(7) M. S. Shaikh, Nonlinear Time Series Analysis Using Time Delay Neural Network, Dissertation for Master of Engineering in Computer Science and System Engineering, Muroran Institute of Technology, Muroran, Japan, 1999.

(8) M. S. Shaikh and Y. Dote, "An Empirical Application of Linear Regression Method and FIR Network for Fault Diagnosis in Nonlinear Time Series", Malaysian Journal of Computer Science, University of Malaya, Kuala Lumpur, Malaysia, Vol. 12, No. 2, Dec. 1999, pp. 57-63.

(9) D. C. Montgomery and G. C. Runger, Applied Statistics and Probability for Engineers, John Wiley \& Sons, Inc. New York, 1994, pp. 474-477.

(10) S. Haykin, Neural Networks: A Comprehensive foundation, 2/e, Prentice-Hall, Inc., Upper Saddle River, NJ, 1999, pp. 652-658.

M. Shafique Shaikh (Non-member) received B.Sc. and

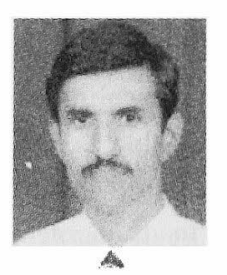

M. Sc. degrees in Electronics and Computer Technology from Univer sity of Sind, Pakistan, in 1983 and 1984, respectively. From 1985 to 1995 he worked in a Pakistan's space agency. He completed his M.Eng. in Computer Science and Systems

Engineering, at Muroran Institute of Technology, Muroran, Japan, in 1999. Currently, Shaikh is a D. Eng. candidate at the same institute. His research interests include signal and image processing, neural networks, and control engineering.

Yasuhiko Dote (Non-member) received his BS degree

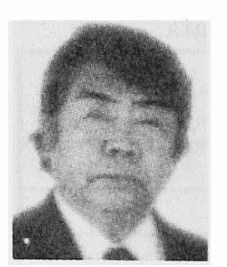
from Muroran Institute of Techno-logy, Muroran, Japan, in 1963. He completed the M. S. and $\mathrm{Ph} . \mathrm{D}$. at University of Missouri, Columbia, in 1972 and 1974 , respectively. From 1963 to 1973 , he has worked in Yaskawa Electric

Manufacturing Co., Ltd., Japan. Presently, Dr. Dote is a Professor in Division of Computer Science and Systems Engineering, Muroran Institute of Technology, Japan. He has many research papers to his credit and has authored and co-authored three books. $\mathrm{He}$ also has organized/chaired many national and international conferences. Dr. Dote's research interests include intelligent control, soft computing, and power electronics. 